\title{
Generation of DNA-based Markers in Specific Genome Regions by Two-primer RAPD Reactions
}

\author{
J. Hu, J. van Eysden, ${ }^{1}$ and C.F. Quiros \\ Department of Vegetable Crops, University of California, Davis, California 95616; ${ }^{1}$ Department of Plant Breeding, Wageningen \\ Agricultural University, The Netherlands
}

Random amplified polymorphic DNA (RAPD) markers offer quick screening of different regions of the genome for genetic polymorphisms. The standard RAPD procedure uses a single 10-base-long random oligonucleotide as a primer to amplify short stretches of the genome by PCR. We modified the procedure by using two primers in each reaction in a Brassica napus mapping project. We found that the two-primer RAPD tends to amplify more and smaller fragments than the standard RAPD technique. These new bands were always ampllfied in the two-primer reactions, and Southern analysis revealed that they had no homology to the bands amplified in single-primer reactions involving the same primers. Furthermore, these new markers were not linked to markers amplified with the same primers in the standard RAPD reactions, suggesting that they were amplified from different genomic regions. The advantage of the twoprimer RAPDs is that it allows more reactions to be carried out with a limited number of primers to generate more markers. Using a single primer, the number of reactions is equal to the number of primers $(n)$, which in turn limits the total number of markers. When using two primers in all possible combinations, the total number of reactions increases to $n \times(n-1 / 2)$. This method could be useful in conjunction with bulked segregant analysis to develop high density maps of certain chromosomal regions. We used this approach to map a second marker linked to a gene governing low linolenic acid concentration in a $B$. napus $F_{2}$ population.

he technique of random amplified polymorphic DNA (RAPD) markers allows quick screening of different regions of the genome for genetic polymorphisms. ${ }^{(1)}$ and is a useful tool for chromosome mapping, ${ }^{(2,3)}$ permitting the location of markers linked to genes of agronomic importance $e^{(4,5)}$ and mapbased cloning of these genes. For example, the Arabidopsis thaliana omega-3 desaturase gene fad 3 and the disease resistance gene RPS2 have been cloned by this approach. ${ }^{(6,7)} \mathrm{A}$ prerequisite for map-based cloning is the development of a highly saturated map in the region containing the gene of interest. Then, the markers close to this gene can be used as starting points for chromosomal walking and eventually finding and cloning the target gene.

We have used RAPDs and restriction fragment-length polymorphisms (RFLPs) for the detection of markers associated with genes determining linolenic acid content in Brassica napus, a trait that seems to be determined by three genes. ${ }^{(8,9)}$ We identified RAPD marker K01-1100 associated with one of these genes. This marker, when converted to a codominant RFLP, accounts for $27 \%$ of the variation for linolenic acid content in our segregating population. ${ }^{(9)} \mathrm{A}$ problem that we encountered in this project was the low level of polymorphism between the parents used to generate the population segregating for seed fatty acid, because of their close genetic proximity. In our quest for additional markers, we tested approximately five hundred 10-nucleotide primers without success. In the search for new polymorphic markers in this population we used two primers in the reactions and found that new and stable markers were generated in the two-primer reactions. In this paper we report the development of a two-primer RAPD technique and its application in identifying markers in specific chromosomal regions, in this case, a region affecting linolenic acid content in a $B$. napus segregating population.

\section{MATERIALS AND METHODS}

In this study, we used an $F_{2}$ population

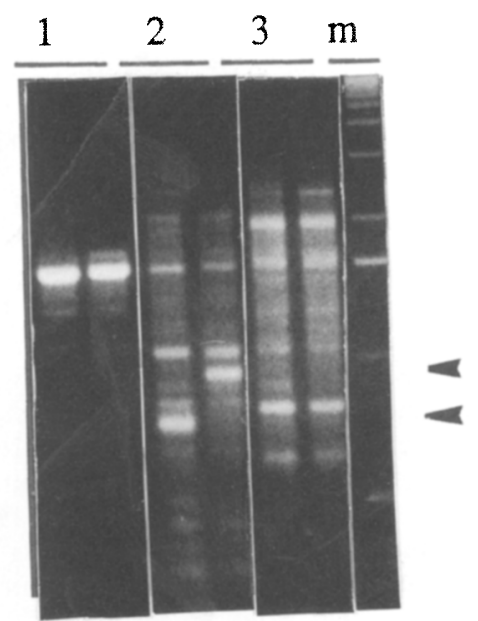

FIGURE 1 Example of polymorphism between two $B$. napus lines run side by side, detectable only by a two-primer RAPD reaction. $(1,3)$ Single-primer amplification profiles for A05 and A06, respectively; (2) two-primer profile $(\mathrm{A} 05+\mathrm{A} 06)$ displaying two polymorphic bands (arrowheads). 


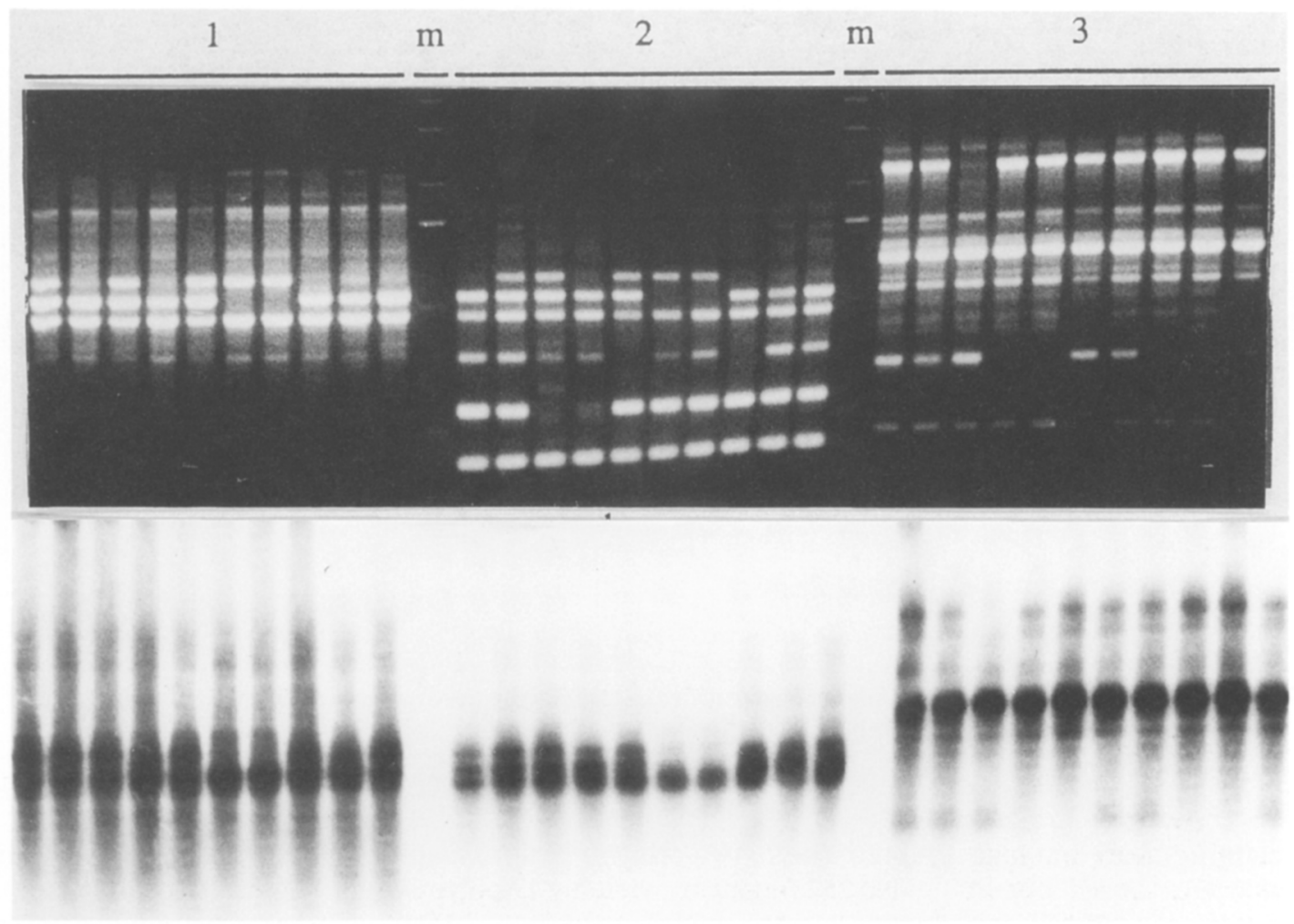

FIGURE 2 Gel hybridization tests for polymorphic bands amplified from selected plants in the B. napus segregating population with single-primer (1) and two-primer reactions. Primer P09; (3) primer 06; (2) P09+ P06 reactions. (Top) Amplification profiles on agarose gel; (bottom) Hybridization of gel with P09 and P06 single-primer total products used as probes. Novel bands in 2 fail to hybridize to these products.

consisting of 126 individuals of rapeseed, B. napus. Genomic DNA was extracted from 3- to 4-week-old $F_{3}$ seedlings following the procedure of $\mathrm{Hu}$ et al. ${ }^{(9)}$ For DNA amplification of twoprimer RAPDs, the protocol of $\mathrm{Hu}$ and Quiros $^{(10)}$ was modified as follows: The reaction mixture consisted of $10 \mathrm{ng}$ of DNA, $25 \mathrm{~mm}$ of each primer (Operon Technologies), $200 \mathrm{~mm}$ each of dATP, dCTP, dGTP, and dTTP (Boehringer Mannheim), $1.9 \mathrm{mM}$ of $\mathrm{MgCl}_{2}, 50 \mathrm{~mm}$
$\mathrm{KCl}, 10 \mathrm{~mm}$ Tris- $\mathrm{HCl}\left(\mathrm{pH} 9.0\right.$ at $25^{\circ} \mathrm{C}$, $0.1 \%$ Triton X-100 and 1 unit of Taq DNA polymerase (Promega). The reaction (10 $\mu \mathrm{l}$, final volume) was covered with two drops of mineral oil in a $0.5-\mathrm{ml}$ microcentrifuge tube. Primer combinations were chosen at random. A Perkin-Elmer Cetus DNA thermal cycler was used to amplify the DNA fragments. The cycler was programmed as follows: one cycle at $94^{\circ} \mathrm{C}$ for $30 \mathrm{sec}$ followed by 45 cycles of $30 \mathrm{sec}$ at $92^{\circ} \mathrm{C}, 1 \mathrm{~min}$ at $35^{\circ} \mathrm{C}$, and $1 \mathrm{~min}$

TABLE 1 Segregation and the Seven Markers Amplified in One and Two-primer Reactions with Primers P05, P06, and P09

\begin{tabular}{|c|c|c|c|c|c|}
\hline \multirow[b]{2}{*}{ Markers } & \multirow{2}{*}{$\begin{array}{l}\text { No. of } F_{2} \\
\text { plants }\end{array}$} & \multicolumn{2}{|c|}{$\begin{array}{l}\text { No. of } F_{2} \text { plants in } \\
\text { genotypic classes }\end{array}$} & \multirow[b]{2}{*}{$x^{2}$} & \multirow[b]{2}{*}{$P$} \\
\hline & & presence & absence & & \\
\hline$P 05+P 09-600$ & 89 & 65 & 24 & 0.18 & $0.50-0.70$ \\
\hline $\mathrm{P} 05+\mathrm{P} 09-800$ & 89 & 63 & 26 & 0.84 & $0.30-0.50$ \\
\hline P05-750 & 93 & 72 & 21 & 0.29 & $0.50-0.70$ \\
\hline $\mathrm{P} 06+\mathrm{P} 09-450$ & 87 & 58 & 29 & 3.22 & $0.05-0.10$ \\
\hline P06-800 & 92 & 63 & 29 & 2.09 & $0.10-0.20$ \\
\hline P09-1100 & 87 & 71 & 16 & 2.03 & $0.10-0.20$ \\
\hline P09-1200 & 90 & 66 & 24 & 0.13 & $0.70-0.80$ \\
\hline
\end{tabular}

at $72^{\circ} \mathrm{C}$ for denaturing, primer annealing, and primer extension, respectively. A final 5 min extension at $72^{\circ} \mathrm{C}$ was carried out after the cycles were completed. The amplified products were separated by electrophoresis on a $2 \%$ agarose gel in $1 \times$ TAE buffer, stained with $10 \mu \mathrm{g} / \mathrm{ml}$ of ethidium bromide, and photographed under UV light with Polaroid black-andwhite film.

Sequence homology of amplified products was determined by direct hybridization on the agarose gels, which were denatured in $0.4 \mathrm{M} \mathrm{NaOH}$ for 10 min and then dried at $80^{\circ} \mathrm{C}$ in a gel dryer. Probes used were either individual fragments or total amplified products of a reaction isolated from agarose gels with a GeneClean Kit (Bio 101, La Jolla, CA). When isolating total amplified products of a reaction, excision of the amplified DNA fragments was done after $10 \mathrm{~min}$ of electrophoresis. The probes were labeled with $\left[{ }^{32} \mathrm{P}\right] \mathrm{dCTP}$ using the Multiprimer er labeling kit (Amersham, Arlington Heights, IL). Hybridization was carried out in $7 \%$ SDS and $250 \mathrm{mM} \mathrm{Na} \mathrm{NPO}_{4}$ at $65^{\circ} \mathrm{C}$ overnight in a hybridization incu- 


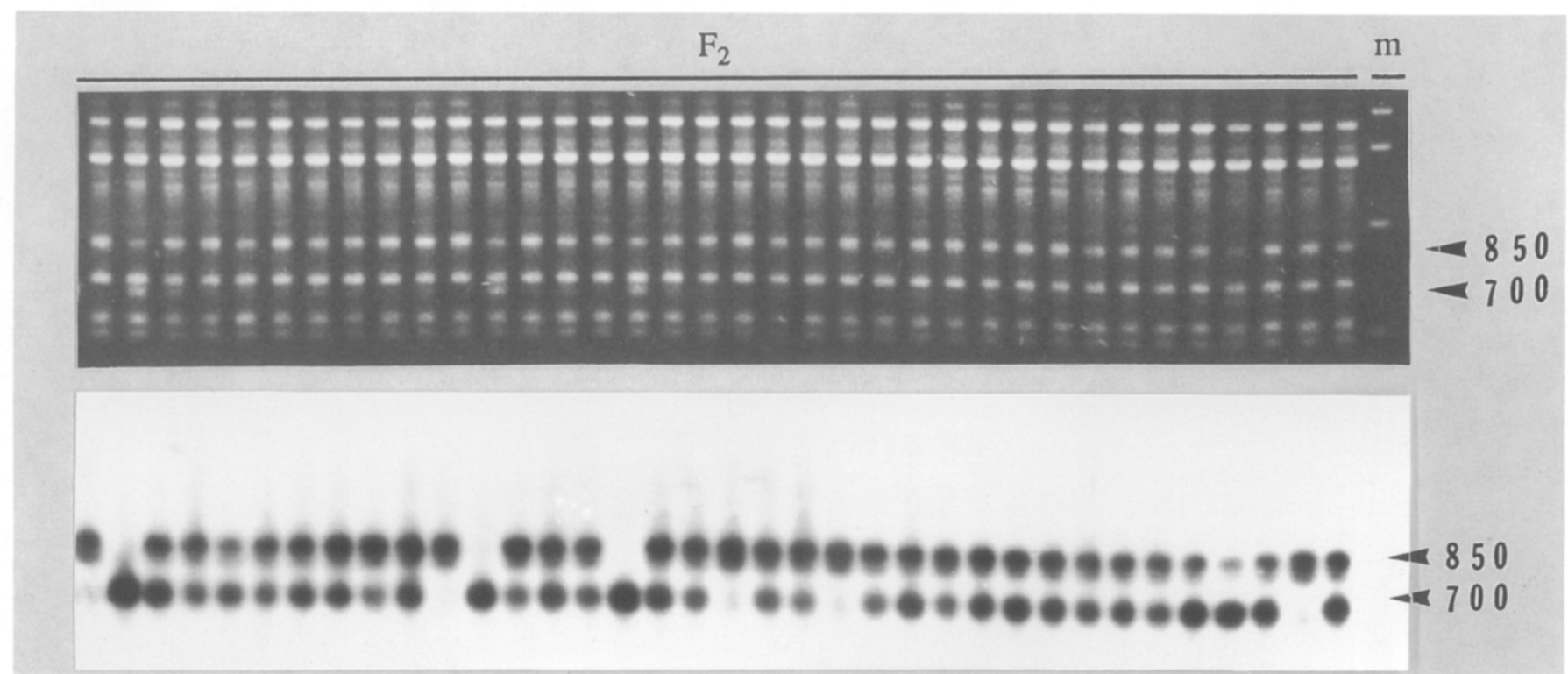

FIGURE 3 Conversion of L01 + L09 RAPD dominant marker segregating 3:1 (top) (arrowheads) to codominant RFLP segregating 1:2:1 (bottom) after gel hybridization with an isolated RAPD band of $650 \mathrm{bp}$.

bator (Robbins Scientific). Low and high stringency washes were carried out at $65^{\circ} \mathrm{C}$ for $30 \mathrm{~min}$ in $5 \%$ SDS and $25 \mathrm{~mm}$ $\mathrm{Na}_{2} \mathrm{HPO}_{4}$, and $1 \%$ SDS and $25 \mathrm{~mm}$ $\mathrm{Na}_{2} \mathrm{HPO}_{4}$, respectively. The gels were than wrapped with Saran Wrap and exposed to X-ray film for 5-12 hr at room temperature.

We used bulked segregant analysis ${ }^{(4)}$ along with two-primer reactions to identify markers associated with linolenic acid concentration. We pooled the DNA samples from $10 \mathrm{~F}_{3}$ plants for each homozygous genotype of marker locus $\mathrm{K} 01-1100$, previously found to be associated with a locus that determines linolenic acid content, ${ }^{(9)}$ and tested them in two-primer RAPDs. DNAs from the two bulked tubes for each genotype and from the two parental tubes were amplified in 140 reactions with 20 RAPD primers, L01-L20. The program MapMaker $^{(11)}$ was used for linkage analysis.

\section{RESULTS AND DISCUSSION}

In the initial experiment, eight primers (A01-A08) were used to amplify DNA from the parental lines of the mapping population in eight single-primer and 28 two-primer RAPD reactions. The latter number of reactions was achieved by all possible combinations of the eight primers. Each reaction gave a unique amplification profile. The eight single-primer RAPD reactions amplified a total of 54 fragments, 7 of which were polymor- phic. The 28 two-primer reactions amplified 252 fragments, yielding 17 polymorphic markers between the two parents. On average, the two-primer RAPDs amplified more bands per reaction than the single-primer RAPDs (9.0 vs. 6.75$)$, but the average size of the amplified bands decreased from 1200 to $950 \mathrm{bp}$. The twoprimer profiles consisted mostly of new bands not seen in the amplification profiles of the single primers. However, these profiles had in common a few bands amplified by one or both primers when run independently. In the singleprimer reactions, primers A03, A04, A05, and A06 did not disclose polymorphism between the two parents. Among the six two-primer reactions generated by four primers, only A05 + A06 amplified two polymorphic bands between the two parents (Fig. 1).

As all the two-primer reactions amplified new, smaller fragments, it was necessary to determine their origin. We found that the new fragments amplified in the two-primer reactions did not hybridize to the amplified products of the single-primer reactions (Fig. 2). However, common-size fragments in the twoprimer reaction hybridized with the single-primer reaction products, indicating that some fragments amplified by one of the primers were also amplified in the two-primer combination. Lack of hybridization of single-primer to twoprimer amplified products of different sizes suggested a lack of homology.
Therefore, the new bands might have originated from genomic regions that were not amplified in the single-primer reactions. This observation was further confirmed by mapping markers generated with a set of three primers in singleand two-primer reactions. A total of seven polymorphic markers were scored with these three primers; four were amplified by single-primer reactions, P05750 , P06-800, P09-1100, and P09-1200, and three by two-primer reactions, P05 + P09-600, P05 + P09-800, and P06 + P09-450. The segregation of each marker fitted the expected 3:1 Mendelian ratio (Table 1). Two markers were mapped on two different linkage groups when tested against 45 RFLP and 51 RAPD loci assigned previously to their respective linkage groups. None of the new markers amplified in the twoprimer reactions was linked to any of the bands amplified by the same primer in the single-primer reaction, thus confirming that each of these markers originated from a different genomic region. These results indicate that certain genomic regions not accessible for single-primer amplification could be reached with the two-primer reactions (see Fig. 5, below).

Using the two-primer RAPD technique, we successfully identified a second marker, L01 + L09-650, linked to a locus affecting linolenic acid content in our rapeseed mapping population. Among the 960 scorable markers amplified in 140 reactions with primer L01- 
L20, one marker (L01+L09-650) was polymorphic between the two bulks, and when tested in the population, segregated in the expected $3: 1$ ratio (100:25; $\left.x^{2}{ }_{3: 1}=1.67, P>0.20\right)$. The plants lacking the 650-bp band generated by this twoprimer combination have lower linolenic acid content. For some plants, the scoring of this band was ambiguous. To resolve this problem, we probed the dry agarose gel containing amplified DNA for these plants with the 650-bp band isolated from the gel. Unexpectedly, it appeared as a codominant locus in the film (Fig. 3, bottom), segregating in a $1: 2: 1 \quad$ ratio $\left(25: 76: 24 ; \quad x_{1: 2: 1}^{2}=5.85\right.$, $P>0.05)$. The alternative allele was $\sim 200$ bp longer and comigrated with another band of similar size; therefore, it could not be scored on the ethidium bromidestained gel (Fig. 3, top). Linkage analysis showed that L01 + L09-650 was linked to $\mathrm{K} 01-1100$ at a distance of $1.6 \mathrm{cM}$. It seems that this second marker is closer to the gene governing linolenic acid than K01-1100, because it accounts for $\sim 32 \%$ of the variation of linolenic acid content in the $\mathrm{F}_{2}$ population. The distribution of linolenic acid content and the marker genotypes revealed by RFLP analysis of $\mathrm{K} 01-1100$ and $\mathrm{L} 01+\mathrm{L} 09-650$ is shown in Figure 4.

Although the use of RAPDs has become widespread, the nature of the amplification is not completely understood because of the constantly changing interactions of the different components through the cyclic reactions. Figure 5 is a schematic representation showing how new RAPD markers may be generated by amplification with the two-primer reactions. A, B, and C, represent different regions in the genome that have randomly distributed sequences homologous to the RAPD primers. Under optimal conditions, the amplification of a fragment is achieved when the primers are bound to the two opposite strands within a distance at which Taq polymerase has extension activity, normally $<4 \mathrm{~kb}$. For example, in two independent singleprimer RAPD reactions, let us assume that primer 1 amplifies a $1.6-\mathrm{kb}$ fragment from region $B$, and primer 2 amplifies two fragments, $1.2 \mathrm{~kb}$ from region $\mathrm{A}$ and $1.4 \mathrm{~kb}$ from region $\mathrm{B}$. It is expected that these two primers will amplify all three

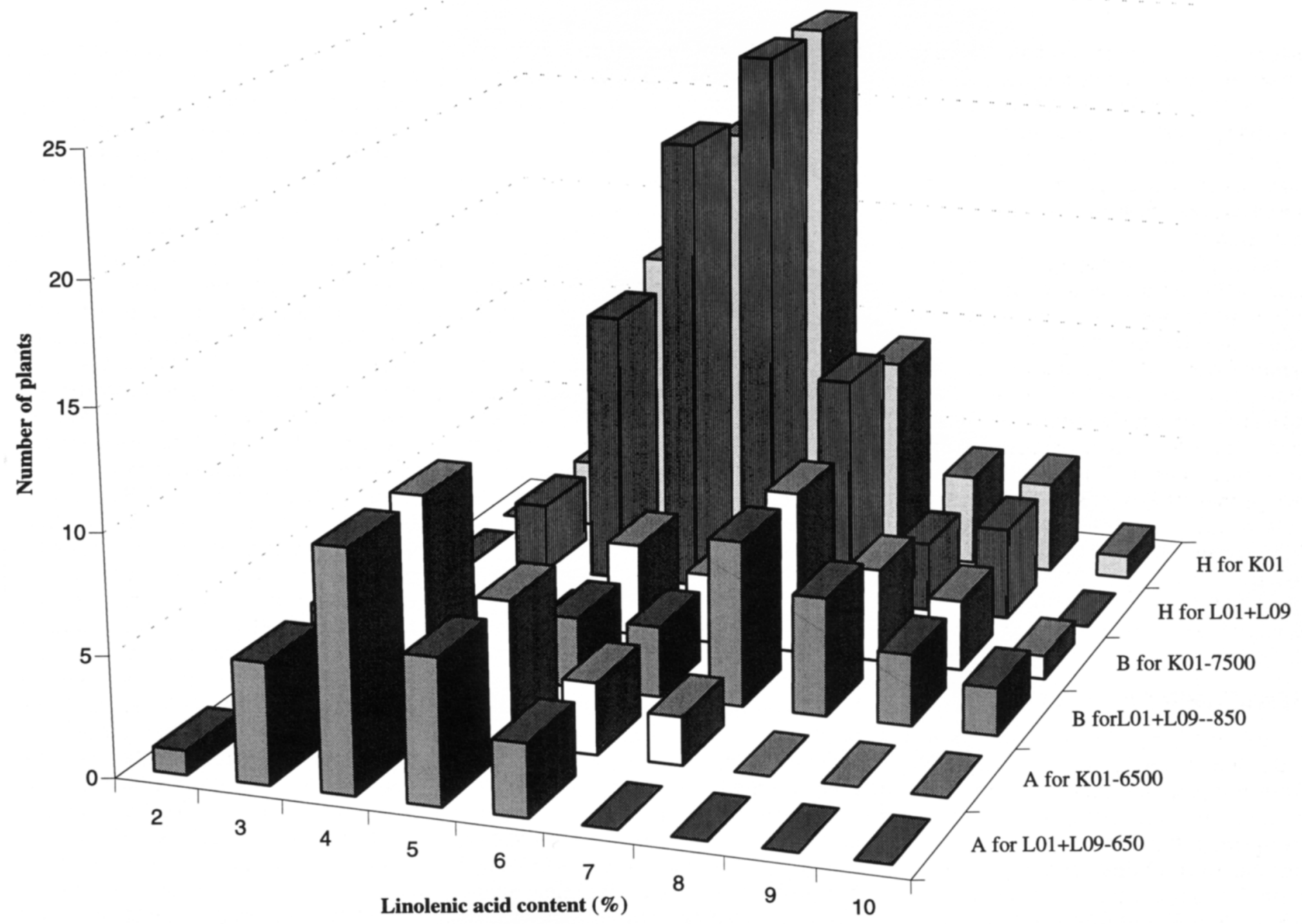

FIGURE 4 Histogram showing cosegregation for linolenic acid content and two codominant markers derived from RAPDs. (First two rows, $A$ ) Homozygous individuals for allele L01 + L09-650 and K01-6500; (middle two rows, $B$ ) homozygous individuals for L01 + L09-850 and K01-7500; (last two rows, $H$ ) heterozygous individuals for L01 + L09 and K01 loci. 
A

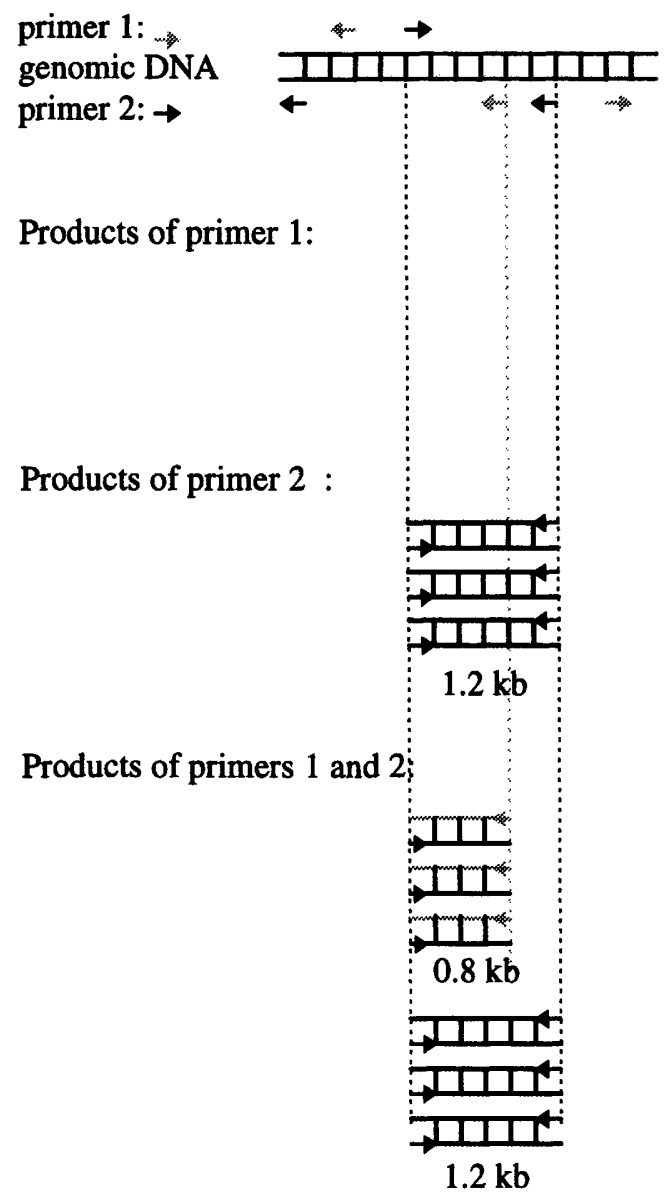

B

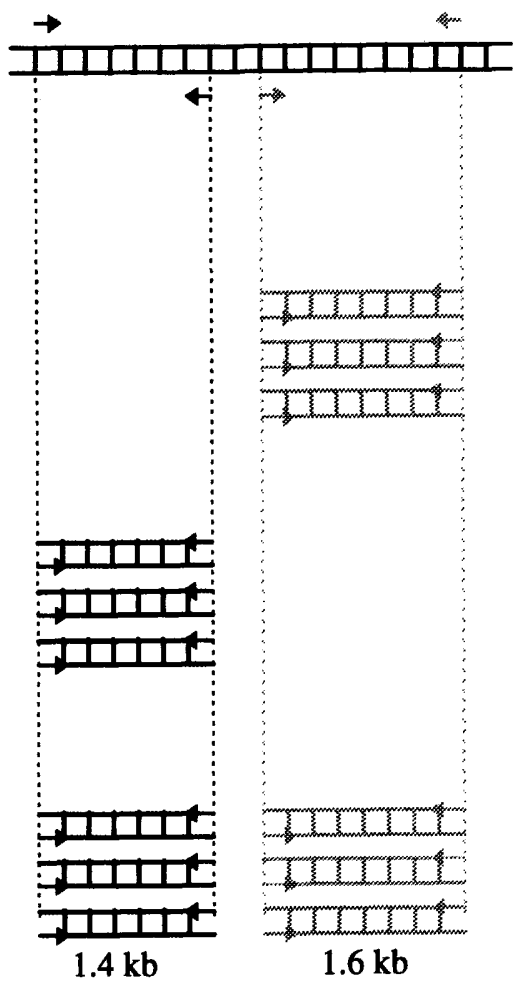

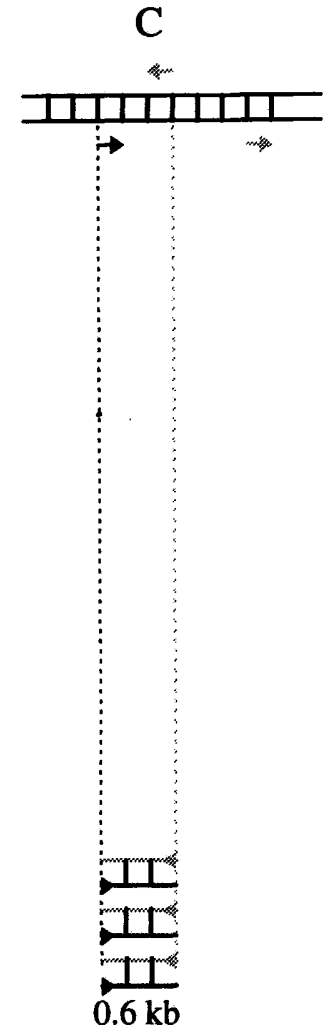

FICURE 5 Schematic representation illustrating origin of novel products by two-primer amplification. $A, B$, and $C$ represent different genomic regions. The light arrow represents primer 1; the dark arrow, primer 2.

fragments if used together in a twoprimer reaction. However, new markers can be generated when the two different primers bind to opposite strands within the amplification range. These can be generated in the same single-primer region of amplification of any of the two primers, such as the 800-bp fragment of region $A$ in Figure 5, as well as anywhere else in the genome, such as the 600-bp fragment in region $C$ in Figure 5 . In our limited testing we observed only the latter case, but it is expected that the former case also occurs, although at very low frequency, resulting from cross-hybridization between overlapping fragments. It should be stated that this sketch is an oversimplification drawn only for illustration purposes. In the actual reactions, the situation is much more complex. We observed that many fragments amplified by one primer were either weaker or even absent from the amplified products of the two-primer re- actions, implying possible competition between the primers. It is difficult to quantify such competition in such a complex system. However, from the probability theory, in the presence of two primers, the new fragments that are amplified by two different primers, one at each end, would be twice as likely to be amplified as those amplified by the same primer at both ends. The coercion of competition varies with primer combinations, resulting in weak to complete absence of certain fragments amplified in single-primer reactions as compared with two-primer reactions containing the same primer.

We have demonstrated the usefulness of the two-primer RAPD method in generating new markers in genome mapping. The advantage of the two-primer RAPD rests on the possibility of increasing the total number of reactions with a limited number of primers. The cost of primers in RAPD reactions is relatively low compared with the other components in the reaction, especially the thermal stable polymerase. However, the total number of primers useful for RAPDs is limited by restrictions on their sequences, which should exclude palindromic and self-annealing sequences, their length, and $\mathrm{G}+\mathrm{C}$ content. Using single primers, the number of reactions is equal to the number of primers $(n)$, which in turn limits the total number of markers. When using two primers together in all possible combinations, the number of reactions will increase to $n \times(n-1 / 2)$. With 1200 RAPD primers, ${ }^{(2)}$ it is possible to set up $>700,000$ reactions. If one reaction on the average amplifies $\sim 8 \mathrm{~kb}$, all of the reactions could theoretically amplify $5.6 \times 10^{9} \mathrm{bp}$, which is almost five haploid genomic equivalents for $B$. napus. Because of its simplicity, the two-primer RAPD should find application in general mapping projects to place markers evenly 
throughout the genome to characterize chromosomes, locate QTLs, and carry out marker-assisted selection. It could also be used to search for polymorphisms, saturate certain genomic regions with the local mapping strategy, ${ }^{(2)}$ generate new markers linked to genes of interest by bulk segregant analysis, ${ }^{(4)}$ and map mutant genes by use of phenotypic pools. ${ }^{(12)}$

\section{ACKNOWLEDGMENTS}

This work was supported by U.S. Department of Agriculture grant 92-37300-7553 to J.H. and C.F.Q. We thank two anonymous reviewers and Dr. R. Michelmore for helpful comments. We are also grateful to Mr. V. D'Antonio, and Ms. L. Goldstein for technical assistance.

\section{REFERENCES}

1. Williams, J.G.K., A.R. Kubelik, K.J. Livak, J.A. Rafalski, and S.V. Tingey. 1990. DNA polymorphisms amplified by arbitrary primers are useful as genetic markers. $\mathrm{Nu}$ cleic Acids Res. 18: 6531-6535.

2. Reiter, R.S., J.G.K. Williams, K.A. Feldmann, J.A. Rafalski, S.V. Tingey, and P.A. Scolnik. 1992. Global and local mapping in Arabidopsis thaliana by using recombinant inbred lines and random amplified polymorphic DNAs. Proc. Natl. Acad. Sci. 89: 1477-1481.

3. Postlethwait, J.H., S.L. Johnson, C.N. Midson, W.S. Talbot, M. Gates, E.W. Ballinger, D. Africa, and R. Andrews. 1994. A genetic linkage map for zebrafish. Science 264: 699-703.

4. Michelmore, R.W., I. Para, and R. Kesselli. 1991. Identification of markers linked to disease-resistance gene by bulked segregant analysis: A rapid method to detect markers in specific genomic regions by using segregation populations. Proc. Natl. Acad. Sci. 88: 9828-9832.

5. Martin, G.B., J.G.K. Williams, and S.D. Tanksley. 1991. Rapid identification of markers linked to a Pseudomonas resistance gene in tomato by using random primers and near-isogenic lines. Proc. Natl. Acad. Sci. 88: 2336-2340.

6. Arondel, V., B. Lemieux, I. Hwang, S. Gibson, H.M. Goodman, and C.R. Somerville. 1992. Map-based cloning of a gene controlling omega-3 fatty acid desaturation in Arabidopsis. Science 258: 1353-1355.

7. Bent, A.F., B.N. Kunkel, D. Dahlbeck, K.L. Brown, R. Schmidt, J. Giraudat, J. Leung, and B.J. Staskawicz. 1994. RPS2 of Arabidopsis thaliana: A leucine-rich repeat class of plant disease resistance gene. Science 265: 1856-1860.
8. Pleines S. and W. Friedt. 1989. Genetic control of LA concentration in seed oil of rapeseed (Brassica napus L.). Theor. Appl. Genet. 78: 793-797.

9. Hu, J., C.F. Quiros, P. Arus, D. Struss, and G. Robbelen. 1995. Mapping of a gene determining linolenic acid content in rapeseed with DNA-based markers. Theor. Appl. Genet. 90: 258-262.

10. Hu, J. and C.F. Quiros. 1991. Identification of broccoli and cauliflower cultivars with RAPD markers. Plant Cell Rep. 10: 505-511.

11. Lincoln, S.E., M.J. Doly, and E.S Lander. 1992. Constructing genetic maps with Mapmaker/Exp 3.0. Whitehead Institute Technical Report, 3rd ed. Whitehead Institute, Cambridge, MA.

12. Williams, J.G.K., R.S. Reiter, R.M. Young, and P.A. Scolnik. 1993. Genetic mapping of mutants using phenotypic pools and mapped RAPD markers. Nucleic Acids Res. 21: $2697-2702$.

Received January 27, 1995; accepted in revised form April 7, 1995. 


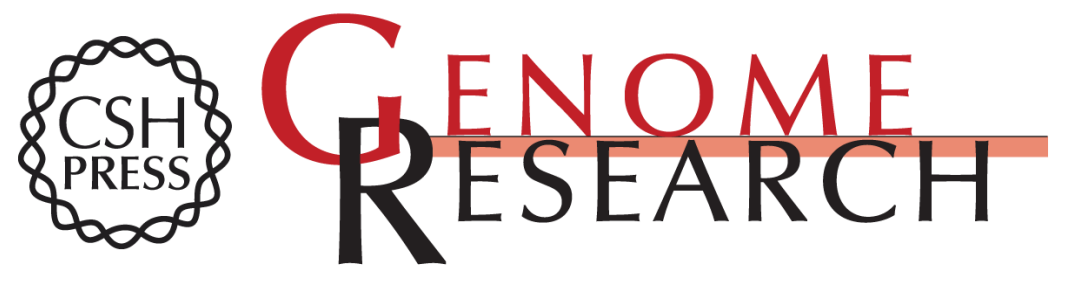

\section{Generation of DNA-based markers in specific genome regions by two-primer RAPD reactions.}

$\mathrm{J} \mathrm{Hu}, \mathrm{J}$ van Eysden and C F Quiros

Genome Res. 1995 4: 346-351

References This article cites 11 articles, 6 of which can be accessed free at: http://genome.cshlp.org/content/4/6/346.full.html\#ref-list-1

License

Email Alerting

Receive free email alerts when new articles cite this article - sign up in the box at the Service top right corner of the article or click here.

\section{Affordable, Accurate Sequencing.}

To subscribe to Genome Research go to: https://genome.cshlp.org/subscriptions 